\title{
Effects of a structured physical activity intervention on measures of physical performance in frail elderly patients after cardiac rehabilitation: a pilot study with 1-year follow-up
}

\author{
Raffaele Molino-Lova • Guido Pasquini - Federica Vannetti • \\ Anita Paperini - Tatiana Forconi · Paola Polcaro • \\ Renato Zipoli $\cdot$ Francesca Cecchi $\cdot$ Claudio Macchi
}

Received: 22 April $2011 /$ Accepted: 19 June 2011

(C) SIMI 2011

\begin{abstract}
The objective of this prospective randomized controlled study was to compare the long-term effects of a structured physical activity intervention with those of aerobic exercises alone, in a cohort of elderly patients who had undergone elective cardiac surgery, and who were classified as frail at the end of rehabilitation based on their Short Physical Performance Battery (SPPB) score. At the end of rehabilitation, 140 frail elderly patients were randomly allocated either to the intervention group (IG) or to the control group (CG). CG participants received the usual aerobic exercise prescription, while IG participants were also taught additional exercises for strength, flexibility, balance and coordination. The improvement in SPPB score after 1 year was the outcome of the study. IG showed a significant improvement in SPPB score $(9.0 \pm 1.1$ vs. $7.7 \pm 1.4, p<0.001)$, while no significant change was found in CG $(7.7 \pm 1.6$ vs. $7.6 \pm 1.5, p=0.252)$. IG also showed a significantly higher proportion of participants who improved their SPPB score of at least 1 point (70 vs. $37 \%, p<0.001)$. In conclusions, our structured physical activity intervention significantly improves the SPPB score in frail elderly patients who have undergone elective cardiac surgery. An intervention that improves the SPPB score might delay the occurrence of mobility disability.
\end{abstract}

Keywords Disability · Frailty · Elderly patients . Cardiac surgery $\cdot$ Rehabilitation

R. Molino-Lova ( $\varangle)$ · G. Pasquini · F. Vannetti - A. Paperini · T. Forconi - P. Polcaro - R. Zipoli - F. Cecchi - C. Macchi Cardiac Rehabilitation Unit, Don Gnocchi Foundation, Via Imprunetana 124, 50023 Pozzolatico, Florence, Italy e-mail: raffmoli@tin.it; rmolino@dongnocchi.it

\section{Introduction}

Disability is the loss of independence in activities of daily living [1]. Although it may occur as a consequence of catastrophic events, in most older persons, disability progresses through a pattern of gradual decline in multiple domains of the physiologic reserve [2, 3], including strength, flexibility, balance and coordination [4]. The clinical condition that precedes the occurrence of disability has been defined as "frailty" [5] and characterizes nondisabled older persons at high risk of incident disability [6].

The Short Physical Performance Battery (SPPB) is a standardized measure of lower extremity physical performance that includes walking, balance and strength tasks [7]. As a low SPPB score is a strong predictor of disability in nondisabled older persons [8], independent of other health conditions and socioeconomic factors [9], the SPPB has been widely used in geriatric research and epidemiological studies to identify frail older persons $[10,11]$. Interestingly, the LIFE-P study [11] provides conclusive evidence that structured physical activity interventions improve the 400-m walking speed and the SPPB score in frail, home dwelling older persons, suggesting that structured physical activity interventions might have the potential to counteract, or at least to slow down, the occurrence of disability.

In a previous study on patients who had undergone cardiac surgery, and who, subsequently, received postacute rehabilitation, we found that two out of three patients were still capable of recovering, or even increasing, their regular physical activity in the year after rehabilitation [12]. Of note, the self-reported level of physical activity was significantly associated with the objective finding of the distance walked at the 6-min walk test. However, in that study, according to current guidelines on cardiac 
rehabilitation, the physical exercise prescription to be performed at home mainly consisted of aerobic exercises $[13,14]$. Further, possible changes in SPPB score over time were not assessed.

As the number of older persons presenting for cardiac surgery is constantly increasing $[15,16]$, understanding whether specific exercises for strength, flexibility, balance and coordination might be successfully integrated into the usual post-rehabilitation exercise prescription, to counteract, or at least to slow down, the gradual decline in physical function in frail older persons, is a relevant issue.

This paper presents a randomized controlled study on nondisabled elderly patients who had undergone elective cardiac surgery and who were classified as frail at the end of rehabilitation, based upon their SPPB score. Our objective was to compare the long-term effects of a structured physical activity intervention that included, along with aerobic training, also specific exercises for strength, flexibility, balance and coordination, with those of aerobic exercises alone. The improvement in SPPB score 1-year after the rehabilitation was the outcome of the study.

\section{Methods}

\section{Study design}

This study was designed as a prospective, parallel-group, pragmatic, randomized controlled study, with 1-year follow-up and $50 \%$ allocation ratio.

\section{Participants}

All nondisabled patients who completed inpatient postacute rehabilitation after elective cardiac surgery were considered eligible for the study. Inclusion criteria were having an age of 65 years or more, and being classified as frail at the end of rehabilitation, based on an SPPB score equal or lower than 9 (on a 0-12 scale, see below) [7]. Patients showing cognitive deterioration (Mini Mental State Examination score equal or lower than 18) [17] or severe depression (15-item Geriatric Depression Scale higher than 10) [18] were excluded from the study. Eligible patients meeting the above criteria were invited to participate in the study, and were asked for their written consent. The Institutional Review Board of the Don Gnocchi Foundation approved the study protocol.

\section{Intervention}

A few days before discharge, patients were randomly allocated (see below) either to the intervention group (IG) or to the control group (CG). CG participants received the usual aerobic exercise prescription [19, 20]. IG participants were also taught, in two extra meetings, additional specific exercises for strength, flexibility, balance and coordination, by two therapists with university degrees in both motor sciences and physiotherapy, and at least 5 years of experience.

Specifically, additional exercises were divided into four groups:

- Exercises aimed at improving upper and lower limb muscle strength. For the upper limb, exercises were designed to increase the strength of shoulder flexors and abductors and elbow flexors. These exercises were performed using additional weights, starting from $0.5 \mathrm{~kg}$ (corresponding to a half-liter bottle of mineral water), with possible increase of $0.5 \mathrm{~kg}$ steps, till $2 \mathrm{~kg}$ max. For the lower limb, exercises were designed to increase the strength of quadriceps, gluteus muscles, hamstrings and gastrocnemius muscles. These exercises were performed without additional weights and changes in the starting position and in the range of motion increased their intensity.

- Exercises aimed at improving body flexibility and muscle length. Along with stretching exercise for back and lower limbs, exercises for neck and shoulder flexibility were also included.

- Exercises aimed at improving dynamic balance. In these exercises patients walked following a straight line with their feet side by side and subsequently in semi tandem and tandem position.

- Exercises aimed at improving coordination. Simple upper and lower limb movements were chosen that gave the possibility to adapt exercise difficulty to the level of coordination shown by each participant. Changing the plane of movements for each limb and, subsequently, combining upper and lower limb movements increased exercise difficulty.

At the end of the two extra meetings, participants were given a booklet with the images of additional exercises, along with their progressive steps, to be performed at home at least three times a week for about $30 \mathrm{~min}$. Participants also received extensive information on the risk of disability, along with its consequences on quality of life and family burden, and were strongly encouraged to continue the above exercises.

\section{Outcome}

The SPPB involves three timed measures of lower extremity performance: balance, five repeated chair stands and 4-m self-paced walking speed [7]. Performance in each of these tasks is assigned an ordinal score ranging from 0 to 4 , with 0 indicating inability to perform the test and 4 the 
highest level of performance. A summary score ranging from 0 (worst performers) to 12 (best performers) is calculated by simply adding walking speed, chair stands and balance scores [7]. Patients showing a summary score equal or lower than 9 are considered as frail, i.e. at high risk for incident disability [6-9].

SPPB score was assessed both at baseline and at 1-year follow-up by the same expert physiotherapist. We regarded as clinically relevant a difference of the SPPB score equal or greater than 1 point, which is considered a substantial change in the pertinent literature [21, 22].

\section{Other variables}

Family history of cardiovascular diseases was ascertained based on the report of death or morbidity for angina, myocardial infarction, stroke or peripheral artery disease in one or both parents, or in one or more siblings, at the age under 65 years. Modifiable cardiovascular risk factors, such as smoking habit, hypertension, diabetes and dyslipidemia were considered categorical variables and ascertained using standard criteria [14]. Body mass index (BMI) was calculated according to the formula: $\mathrm{BMI}=$ weight $(\mathrm{kg}) /$ height $(\mathrm{m})^{2}$. All patients underwent echocardiography, using a My Lab 30 apparatus (ESAOTE, Genoa, Italy) equipped with a $2.5 \mathrm{MHz}$ imaging transducer and left ventricular ejection fraction was assessed using standardized criteria [23]. Chronic atrial fibrillation was also ascertained using standardized criteria. The 6-min walk test was performed according to the recommendations of the American Thoracic Society [24].

\section{Sample size}

The sample size was estimated based on a large series of nondisabled elderly patients who had undergone SPPB score assessment at the end of the rehabilitation in our center. As the mean SPPB score among those who showed a score equal or lower than 9 was, consistently with previously reported data [11], 7.6 \pm SD 1.4, we estimated that a sample of around 45 participants per group would have been able to detect a difference of at least 1 point in the SPPB score at follow-up, with a 0.9 power at the two-sided 0.05 level. However, taking into consideration both a possible low attendance rate of the follow-up visit and a possible low adherence rate to the exercise prescription, we enrolled 70 participants per group.

\section{Randomization}

Once enrolled, each participant was given a progressive number and the sister of the ward assigned patients to their group based upon a two-column series (1 for each group) of randomly generated numbers, without any restriction.

\section{Blinding}

The physiotherapist who performed both baseline and follow-up SPPB assessment was blind as to which group the patient had been assigned.

\section{Statistics}

Data are reported as mean \pm standard deviation or as absolute number, along with the percentage in brackets. Statistical analyses were performed using the STATA 7.0 software, from Stata Corporation (College Station, TX, USA). Differences in baseline characteristics between groups were tested using the Student's $t$ test or the Pearson's $\chi^{2}$ test, for continuous or categorical variables, respectively. Differences in SPPB scores between groups, both at baseline and at follow-up, were tested using the Kruskal-Wallis rank test, and differences from baseline to follow-up were tested using the Wilcoxon's rank test. Then, changes in SPPB score from baseline to follow-up were classed into three categories (improvement of at least 1 point, unchanged and worsening) and risk differences between groups, along with risk ratios, were calculated using the Fisher exact test. Finally, for ancillary analyses, we also dichotomized both baseline SPPB score (4-6 vs. 7-9) and follow-up SPPB score (equal or lower than 9 vs. higher than 9), and risk differences and ratios were also calculated using the Fisher exact test.

\section{Results}

Participant flow

Figure 1 shows the flow diagram of the study. We assessed for eligibility 293 nondisabled consecutive patients who had undergone elective cardiac surgery and who completed post-acute inpatient rehabilitation: 128 patients did not meet the inclusion criteria, 55 (19\% of eligible patients) because the age was under 65 years and 73 (31\% of eligible patients aged 65 years or more) because the SPPB score at the end of the rehabilitation was higher than 9 . Eleven patients showed either cognitive deterioration or severe depression and were excluded. Further, 14 patients declined to participate in the study. The remaining 140 patients (93 men and 47 women, mean age 74 years \pm SD 6 , age range $65-88$, median 73.5 , 1st quartile 69.5 , 3rd quartile 79) were randomly allocated, 70 each group, either to the IG or to the CG. Altogether, the attendance rate of the follow-up visit was $89 \%$ and the self-reported 
Fig. 1 Flow diagram of the study

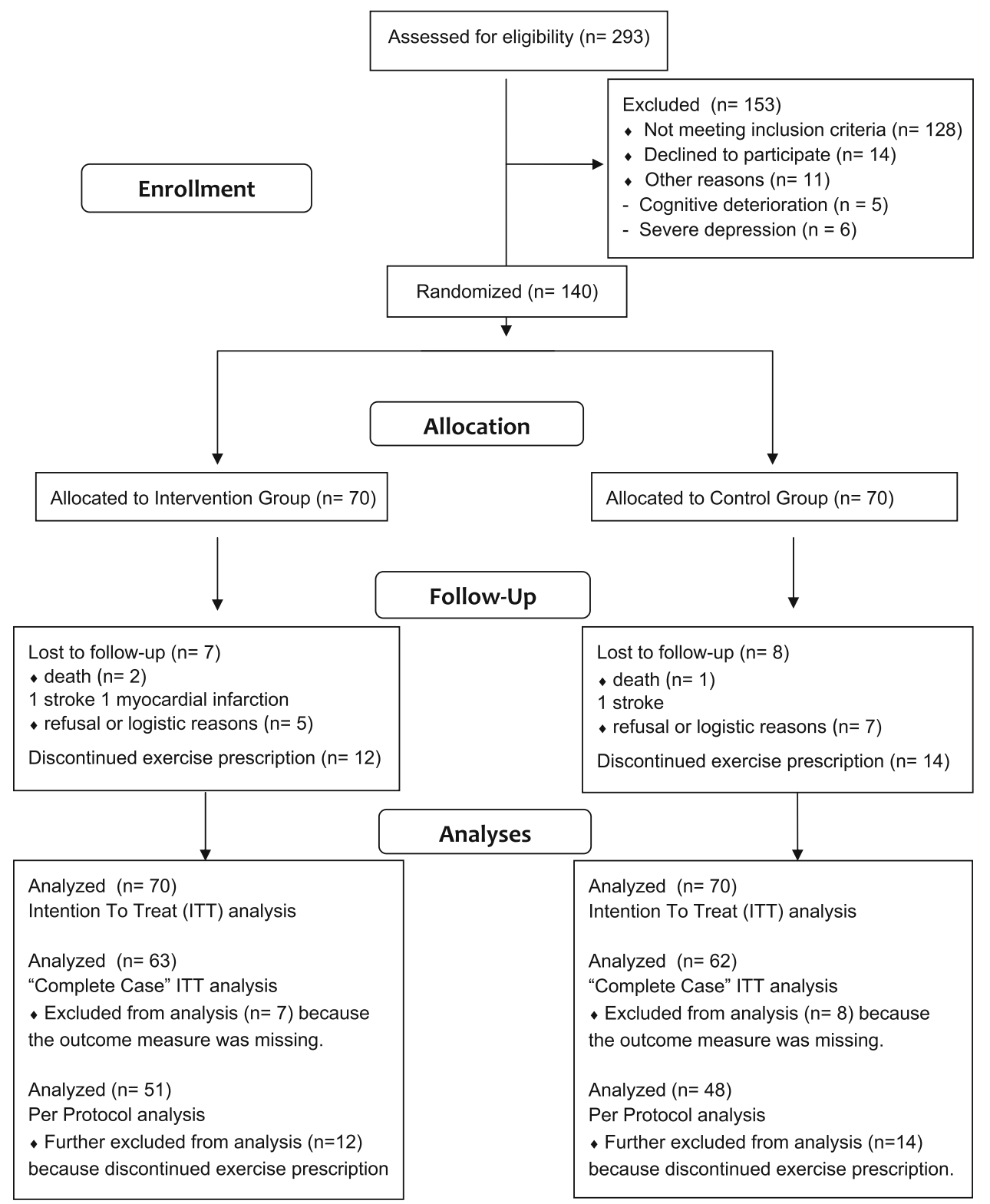

adherence rate to the physical exercise prescription for both structured intervention and aerobic exercise alone was around $70 \%$.

\section{Recruitment}

Participants were recruited from May 2008 to 2009 and were invited for the follow-up visit 1 year after discharge, from May 2009 to 2010.

Baseline data

Baseline demographic and clinical characteristics of study participants according to randomized groups are shown in Table 1. No significant difference was found between IG and CG. Baseline SPPB score ranged from 4 to 9 in both IG and CG (Table 2) and scores were evenly distributed in the two groups.

Number analyzed

Intention to treat analysis (ITT), with the original number of participants (70 each group, $n=140$ ) as denominator, was first performed. Then, as 15 participants were lost to follow-up and the outcome measure was no way available, instead of using imputation techniques, we performed the "complete case" ITT [25], using as denominator the number of participants who attended the follow-up visit (63 in IG and 62 in CG, $n=125$ ). Finally, as further 26 participants discontinued the exercise prescription, we also 
Table 1 Baseline characteristics of study participants according to randomized groups $(n=140)$

* From two-tailed Student's $t$ test or Pearson's $\chi^{2}$ test, as appropriate

Table 2 Changes in Short Physical Performance Battery (SPPB) score according to randomized groups in participants who completed the follow-up $(n=125)$
* From Kruskal-Wallis rank test

** From Wilcoxon rank test

\begin{tabular}{|c|c|c|c|}
\hline & $\begin{array}{l}\text { Intervention group } \\
(n=70)\end{array}$ & $\begin{array}{l}\text { Control group } \\
(n=70)\end{array}$ & $p^{*}$ \\
\hline \multicolumn{4}{|l|}{ Demographics } \\
\hline Age, years (mean $\pm \mathrm{SD})$ & $74.7 \pm 6.3$ & $73.9 \pm 6.0$ & 0.443 \\
\hline Female sex, $n(\%)$ & $19(27)$ & $28(40)$ & 0.107 \\
\hline \multicolumn{4}{|l|}{ Operation } \\
\hline Coronary artery by-pass graft, $n(\%)$ & $22(31)$ & $30(43)$ & \\
\hline Valve repair/replacement, $n(\%)$ & $30(43)$ & $24(34)$ & 0.365 \\
\hline Combined procedures, $n(\%)$ & $18(26)$ & $16(23)$ & \\
\hline \multicolumn{4}{|l|}{ Cardiovascular risk factors } \\
\hline Family history of cardiovascular diseases, $n(\%)$ & $20(29)$ & $18(26)$ & 0.824 \\
\hline Current smokers, $n(\%)$ & $4(6)$ & $1(1.5)$ & 0.188 \\
\hline Hypertension, $n(\%)$ & $51(73)$ & $43(61)$ & 0.225 \\
\hline Diabetes, $n(\%)$ & $13(19)$ & $15(21)$ & 0.580 \\
\hline Dyslipidemia, $n(\%)$ & $15(21)$ & $14(20)$ & 0.939 \\
\hline Body mass index $\left(\mathrm{kg} / \mathrm{m}^{2}\right)($ mean $\pm \mathrm{SD})$ & $25.3 \pm 3.0$ & $25.7 \pm 2.8$ & 0.486 \\
\hline Left ventricular ejection fraction, $\%$ (mean \pm SD) & $52.4 \pm 9.1$ & $52.6 \pm 8.7$ & 0.901 \\
\hline Chronic atrial fibrillation, $n(\%)$ & $5(7)$ & $7(10)$ & 0.546 \\
\hline 6-min walk test, $m$ (mean $\pm \mathrm{SD})$ & $334 \pm 68$ & $329 \pm 72$ & 0.719 \\
\hline
\end{tabular}

\begin{tabular}{|c|c|c|c|}
\hline & $\begin{array}{l}\text { Intervention group } \\
(n=63)\end{array}$ & $\begin{array}{l}\text { Control group, } \\
(n=62)\end{array}$ & $p^{*}$ \\
\hline \multicolumn{4}{|l|}{ Baseline SPPB score } \\
\hline Score $4, n(\%)$ & $1(2)$ & $2(3)$ & \\
\hline Score $5, n(\%)$ & $7(11)$ & $7(11)$ & \\
\hline Score $6, n(\%)$ & $3(5)$ & $3(5)$ & \\
\hline Score $7, n(\%)$ & $11(17)$ & $12(19)$ & 0.259 \\
\hline Score $8, n(\%)$ & $15(24)$ & 17 (27) & \\
\hline Score $9, n(\%)$ & $26(41)$ & $21(34)$ & \\
\hline Mean score $( \pm \mathrm{SD})$ & $7.7 \pm 1.4$ & $7.6 \pm 1.5$ & \\
\hline \multicolumn{4}{|l|}{ Follow-up SPPB score } \\
\hline Score $4, n(\%)$ & $0(0)$ & $1(2)$ & \\
\hline Score $5, n(\%)$ & $0(0)$ & $7(11)$ & \\
\hline Score $6, n(\%)$ & $2(3)$ & $5(8)$ & \\
\hline Score $7, n(\%)$ & $5(8)$ & 12 (19) & \\
\hline Score $8, n(\%)$ & $7(11)$ & $13(21)$ & $<0.001$ \\
\hline Score $9, n(\%)$ & $30(48)$ & $20(32)$ & \\
\hline Score $10, n(\%)$ & $13(20.5)$ & $3(5)$ & \\
\hline Score $11, n(\%)$ & $6(9.5)$ & $1(2)$ & \\
\hline Score $12, n(\%)$ & $0(0)$ & $0(0)$ & \\
\hline Mean score $( \pm \mathrm{SD})$ & $9.0 \pm 1.1$ & $7.7 \pm 1.6$ & \\
\hline$p^{* *}$ & $<0.001$ & 0.252 & \\
\hline
\end{tabular}

performed the per protocol analysis (PP), using as denominator the number of participants who both attended the follow-up visit and continued the exercise prescription (51 in IG and 48 in CG, $n=99$ ).
Outcome and estimation

Table 2 shows changes in SPPB score according to randomized groups in participants who attended the follow-up 
Table 3 Changes in Short Physical Performance Battery (SPPB) score according to randomized groups: primary analyses

\begin{tabular}{|c|c|c|c|c|c|}
\hline & Intervention group & Control group & $p^{*}$ & Risk difference (95\% CI) & Risk ratio $(95 \% \mathrm{CI})$ \\
\hline \multicolumn{6}{|l|}{ Intention to treat analysis (ITT) $(n=140)$} \\
\hline SPPB improvement $\geq 1$ point, $n(\%)$ & $44 / 70(63)$ & $23 / 70(33)$ & $<0.001$ & $30 \%(14-46)$ & $1.91(1.31-2.80)$ \\
\hline SPPB unchanged, $n(\%)$ & $18 / 70(26)$ & $24 / 70(34)$ & 0.269 & $-9 \%(-24$ to 7$)$ & $0.75(0.45-1.25)$ \\
\hline SPPB worsening $\geq 1$ point, $n(\%)$ & $1 / 70(1)$ & $15 / 70(21)$ & $<0.001$ & $-20 \%(-30$ to -10$)$ & $0.07(0.01-0.49)$ \\
\hline \multicolumn{6}{|l|}{ "Complete case" ITT analysis $(n=125)$} \\
\hline SPPB improvement $\geq 1$ point, $n(\%)$ & $44 / 63(70)$ & 23/62 (37) & $<0.001$ & $33 \%(16-49)$ & $1.88(1.31-2.71)$ \\
\hline SPPB unchanged, $n(\%)$ & $18 / 63(28.5)$ & $24 / 62(39)$ & 0.260 & $-10 \%(-26$ to 6$)$ & $0.74(0.45-1.22)$ \\
\hline SPPB worsening $\geq 1$ point, $n(\%)$ & $1 / 63(1.5)$ & $15 / 62(24)$ & $<0.001$ & $-23 \%(-34$ to -12$)$ & $0.07(0.01-0.48)$ \\
\hline \multicolumn{6}{|l|}{ Per protocol analysis $(n=99)$} \\
\hline SPPB improvement $\geq 1$ point, $n(\%)$ & $41 / 51(80)$ & $18 / 48(38)$ & $<0.001$ & $42 \%(25-60)$ & $2.14(1.52-3.16)$ \\
\hline SPPB unchanged, $n(\%)$ & $10 / 51(20)$ & $20 / 48(41)$ & 0.017 & $-21 \%(-40$ to -4$)$ & $0.66(0.38-1.15)$ \\
\hline SPPB worsening $\geq 1$ point, $n(\%)$ & $0 / 51(0)$ & $10 / 48(21)$ & $<0.001$ & $-21 \%(-32$ to -9$)$ & - \\
\hline
\end{tabular}

* From two-tailed Fisher's exact test

visit. IG participants showed a significant improvement in the SPPB score from baseline to follow-up, while no significant change was found in CG participants. Table 3 shows primary analyses, along with risk differences and ratios. In all analyses, IG showed a significantly higher proportion of participants who improved of at least 1 point, and, conversely, a significantly lower proportion of participants who worsened their SPPB score. In the PP analysis only, IG also showed a significantly lower proportion of participants who maintained their SPPB score unchanged.

\section{Ancillary analyses}

A first set of ancillary exploratory subgroup analyses were performed after having dichotomized the baseline SPPB score. In the subgroup with baseline SPPB score 4-6, IG showed a significantly greater improvement in the SPPB score than $\mathrm{CG}(2.5 \pm 1.4$ vs. $1.2 \pm 1.4, p=0.023)$. Within this subgroup, there was no significant difference in the proportion of participants who either improved or worsened or maintained unchanged their SPPB score (Table 4). In the subgroup with baseline SPPB score 7-9, IG also showed a significantly greater improvement in the SPPB score than CG $(1.0 \pm 1.1$ vs. $-0.1 \pm 1.2$, $p<0.001)$. Within this subgroup, IG showed a significantly higher proportion of participants who improved of at least 1 point and, conversely, a significantly lower proportion of participants who worsened their SPPB score. In the per protocol analysis only, IG also showed a significantly lower proportion of participants with unchanged SPPB score (Table 4). A second set of ancillary exploratory subgroup analyses were performed after having dichotomized the follow-up SPPB score. In all analyses, IG showed a significantly higher proportion of participants with a follow-up SPPB score higher than 9 (Table 5), i.e. a significantly higher proportion of participants who were no more classified as frail after 1 year.

\section{Harm}

No clinically relevant adverse event attributable to the physical activity intervention was reported.

\section{Discussion}

In this randomized controlled study on nondisabled elderly patients who had undergone elective cardiac surgery and who were classified as frail at the end of rehabilitation, we compared the long-term effects of a structured physical activity intervention with those of aerobic exercises alone. After 1 year, we found that participants who received the structured physical activity intervention showed a significantly greater improvement in the SPPB score and a significantly higher proportion of participants who improved their SPPB score of at least 1 point.

Limitations

The single-site design and the relatively small number of participants are the main methodological limitations of this study.

\section{Generalizability}

Other inherent limitations of this study may restrict the generalizability of our results. In fact, we selectively investigated a cohort of frail elderly patients who had undergone elective cardiac surgery and who, subsequently, 
Table 4 Changes in Short Physical Performance Battery (SPPB) score according to randomized groups and baseline SPPB scores: ancillary exploratory subgroup analyses

\begin{tabular}{|c|c|c|c|c|c|}
\hline & Intervention group & Control group & $p^{*}$ & Risk difference (95\% CI) & Risk ratio $(95 \% \mathrm{CI})$ \\
\hline \multicolumn{6}{|l|}{ Intention to treat analysis (ITT) $(n=140)$} \\
\hline \multicolumn{6}{|l|}{ Baseline SPPB 4-6 $(n=32)$} \\
\hline SPPB improvement $\geq 1$ point, $n(\%)$ & $10 / 14(71)$ & $9 / 18(50)$ & 0.221 & $21 \%(-12$ to 54$)$ & $1.43(0.81-2.52)$ \\
\hline SPPB unchanged, $n(\%)$ & $1 / 14(7)$ & $1 / 18(5.5)$ & 0.854 & $1.5 \%(-16$ to 19$)$ & $1.29(0.09-18.8)$ \\
\hline SPPB worsening $\geq 1$ point, $n(\%)$ & $0 / 14(0)$ & $2 / 18(11)$ & 0.198 & $-11 \%(-26$ to 3$)$ & - \\
\hline \multicolumn{6}{|l|}{ Baseline SPPB 7-9 $(n=108)$} \\
\hline SPPB improvement $\geq 1$ point, $n(\%)$ & $34 / 56(61)$ & $14 / 52(27)$ & $<0.001$ & $34 \%(16-51)$ & $2.26(1.38-3.70)$ \\
\hline SPPB unchanged, $n(\%)$ & $17 / 56(30)$ & $23 / 52(44)$ & 0.136 & $-14 \%(-32$ to 4$)$ & $0.69(0.42-1.13)$ \\
\hline SPPB worsening $\geq 1$ point, $n(\%)$ & $1 / 56(2)$ & $13 / 52(25)$ & $<0.001$ & $-23 \%(-35$ to -11$)$ & $0.07(0.01-0.53)$ \\
\hline \multicolumn{6}{|l|}{ "Complete case" ITT analysis $(n=125)$} \\
\hline \multicolumn{6}{|l|}{ Baseline SPPB 4-6 $(n=23)$} \\
\hline SPPB improvement $\geq 1$ point, $n(\%)$ & $10 / 11(91)$ & $9 / 12(75)$ & 0.590 & $16 \%(-14$ to 46$)$ & $1.21(0.83-1.77)$ \\
\hline SPPB unchanged, $n(\%)$ & $1 / 11(9)$ & $1 / 12(8)$ & 1.000 & $1 \%(-22$ to 24$)$ & $1.09(0.07-15.4)$ \\
\hline SPPB worsening $\geq 1$ point, $n(\%)$ & $0 / 11(0)$ & 2/12 (17) & 0.478 & $-17 \%(-38$ to 4$)$ & - \\
\hline \multicolumn{6}{|l|}{ Baseline SPPB 7-9 $(n=102)$} \\
\hline SPPB improvement $\geq 1$ point, $n(\%)$ & $34 / 52(65)$ & $14 / 50(28)$ & $<0.001$ & $37 \%(19$ to 55$)$ & $2.34(1.44-3.80)$ \\
\hline SPPB unchanged, $n(\%)$ & $17 / 52(33)$ & $23 / 50(46)$ & 0.224 & $-13 \%(-32$ to 5$)$ & $0.71(0.43-1.16)$ \\
\hline SPPB worsening $\geq 1$ point, $n(\%)$ & $1 / 52(2)$ & $13 / 50(26)$ & $<0.001$ & $-24 \%(-37$ to -11$)$ & $0.07(0.01-0.54)$ \\
\hline \multicolumn{6}{|l|}{ Per protocol analysis $(n=99)$} \\
\hline \multicolumn{6}{|l|}{ Baseline SPPB 4-6 $(n=18)$} \\
\hline SPPB improvement $\geq 1$ point, $n(\%)$ & $9 / 9(100)$ & $7 / 9(78)$ & 0.134 & $22 \%(-5$ to 49$)$ & $1.29(0.91-1.82)$ \\
\hline SPPB unchanged, $n(\%)$ & $0 / 9(0)$ & $0 / 9(0)$ & 1.000 & - & - \\
\hline SPPB worsening $\geq 1$ point, $n(\%)$ & $0 / 9(0)$ & $2 / 9(22)$ & 0.134 & $-22 \%(-49$ to 5$)$ & - \\
\hline \multicolumn{6}{|l|}{ Baseline SPPB 7-9 $(n=81)$} \\
\hline SPPB improvement $\geq 1$ point, $n(\%)$ & $32 / 42(76)$ & $11 / 39(28)$ & $<0.001$ & $48 \%(29-67)$ & $2.70(1.59-4.58)$ \\
\hline SPPB unchanged, $n(\%)$ & $10 / 42(24)$ & $20 / 39(51)$ & 0.011 & $-27 \%(-48$ to -7$)$ & $0.46(0.25-0.86)$ \\
\hline SPPB worsening $\geq 1$ point, $n(\%)$ & $0 / 42(0)$ & $8 / 39(21)$ & 0.002 & $-21 \%(-33$ to -8$)$ & - \\
\hline
\end{tabular}

* From two-tailed Fisher's exact test

Table 5 Changes in Short Physical Performance Battery (SPPB) score according to randomized groups and follow-up SPPB scores: ancillary exploratory subgroup analyses

\begin{tabular}{lccccc}
\hline & Intervention group & Control group & $p^{*}$ & Risk difference (95\% CI) & Risk ratio (95\% CI) \\
\hline Intention to treat analysis (ITT) & $(n=140)$ & & & & \\
$\quad$ Follow-up SPPB $>9, n(\%)$ & $19 / 70(27)$ & $4 / 70(6)$ & 0.001 & $21 \%(10-33)$ & $4.75(1.70-13.3)$ \\
Follow-up SPPB $\leq 9, n(\%)$ & $44 / 70(63)$ & $58 / 70(83)$ & 0.013 & $-20 \%(-34$ to -6$)$ & $0.76(0.62-0.94)$ \\
“Complete case” ITT analysis $(n=125)$ & & & & $4.67(1.69-13.0)$ \\
Follow-up SPPB $>9, n(\%)$ & $19 / 63(30)$ & $4 / 62(6)$ & $<0.001$ & $24 \%(11-37)$ & $0.75(0.63-0.89)$ \\
Follow-up SPPB $\leq 9, n(\%)$ & $44 / 63(70)$ & $58 / 62(94)$ & $<0.001$ & $-24 \%(-37$ to -11$)$ & \\
Per protocol analysis $(n=99)$ & & & & \\
Follow-up SPPB $>9, n(\%)$ & $19 / 51(37)$ & $3 / 48(6)$ & $<0.001$ & $31 \%(16-46)$ & $5.96(1.88-18.9)$ \\
Follow-up SPPB $\leq 9, n(\%)$ & $32 / 51(63)$ & $45 / 48(94)$ & $<0.001$ & $-31 \%(-46$ to -16$)$ & $0.67(0.54-0.84)$ \\
\hline
\end{tabular}

* From two-tailed Fisher's exact test

received post-acute inpatient rehabilitation. Accordingly, our result cannot be directly extended to all frail older persons or to all frail elderly patients with cardiac diseases, either not receiving rehabilitation or receiving outpatient rehabilitation or receiving inpatient rehabilitation for clinical conditions other than elective cardiac surgery. 
Interpretation

To the best of our knowledge, the long-term effects of a structured physical activity intervention on measures of physical performance in nondisabled elderly patients who had undergone elective cardiac surgery and who were classified as frail at the end of rehabilitation have never been reported before. Although direct comparisons with the existing literature are, obviously, not feasible, the LIFE-P study [11], in which the effects of structured physical activity interventions were tested in frail, home dwelling older persons, might, anyhow, be a suitable reference.

In our cohort, both the attendance rate of the follow-up visit and the adherence rate to the physical exercise prescription were quite high (89 and $70 \%$, respectively) and these findings are aligned with the results of both the LIFE$P$ study [11] and a previous study from our group [12].

Although LIFE-P study participants [11] and our elderly patients had similar baseline SPPB scores, after 1 year, our intervention group showed a greater SPPB score improvement and a higher proportion of participants who increased of at least 1 point, or who maintained unchanged, their SPPB score. These findings might be accounted for by the fact that our participants had recently undergone a cardiac operation. Indeed, elective cardiac surgery is aimed at increasing life expectancy, and, which might be even more important for very old patients [26], at improving quality of life and independence through the relief of limiting symptoms, such as chest pain, shortness of breath or dysrhythmias [27]. However, elderly patients frequently show a slower and more complicated recovery after cardiac surgery [12, 13]. In this study, we found that a relevant proportion of patients aged 65 years or more $(69 \%$, see participant flow) were classified as frail at the end of postacute rehabilitation, based on an SPPB score equal or lower than 9. Unluckily, data on preoperative SPPB score were not available, so that we were not able to ascertain either the possible preexisting frailty status or the amount of frailty, if any, that was attributable to the operation. However, it could reasonably be hypothesized that in our elderly patients a certain amount of frailty might have resulted from the operation [28]. This might explain why the improvement in SPPB score was higher in this study than in the LIFE-P study [11], in which participants reporting recent major surgery were temporarily excluded.

Interestingly, ancillary analyses showed that in the subgroup with baseline SPPB score 4-6 the proportion of participants who improved their SPPB score of at least 1 point was not significantly different in intervention and control groups. Although this subgroup was quite small, and hence sample size may well have been underpowered, it might also be hypothesized that participants with SPPB score 4-6 were so functionally impaired that they benefited from any form of physical activity, independent of the type of exercises. Finally, in the intervention group, the proportion of participants who showed a follow-up SPPB score higher than 9 , i.e. the proportion of participants who were no more classified as frail after 1 year, was significantly higher.

In conclusion, our data show that a structured physical activity intervention that includes, along with aerobic training, also specific exercises for strength, flexibility, balance and coordination significantly improves the SPPB score in nondisabled elderly patients who have undergone elective cardiac surgery and who are classified as frail at the end of rehabilitation. Although we investigated a small portion of frail older persons, our findings are relevant because the number of older persons presenting for cardiac surgery is constantly increasing [16]. According to current guidelines on cardiac rehabilitation, these patients at the end of the rehabilitation are given a physical exercise prescription mainly consisting of aerobic exercise for the secondary or tertiary prevention of cardiovascular diseases [14]. The findings of this study suggest that specific exercises for strength, flexibility, balance and coordination may be successfully integrated into the usual post-rehabilitation exercise prescription to counteract, or at least to slow down, the decline in physical function in frail elderly patients.

Acknowledgments Dr. Polcaro and Dr. Forconi were supported by the EnteCassa di Risparmio di Firenze (Contract 2007-0263).

Conflict of interest None.

\section{References}

1. Stucki G, Melvin J (2007) The International Classification of Functioning, Disability and Health: a unifying model for the conceptual description of physical and rehabilitation medicine. J Rehabil Med 39:286-292

2. Ferrucci L, Guralnik JM, Studenski S, Fried LP, Cutler GB Jr, Walston JD (2004) Designing randomized, controlled trials aimed at preventing or delaying functional decline and disability in frail, older persons: a consensus report. J Am Geriatr Soc 52:625-634

3. Bortz WM (2002) A conceptual framework of frailty: a review. J Gerontol A Biol Sci Med Sci 57:M283-M288

4. Fried LP, Ferrucci L, Darer J, Williamson JD, Anderson G (2004) Untangling the concepts of disability, frailty, and comorbidity: implications for improved targeting and care. J Gerontol A Biol Sci Med Sci 59:255-263

5. Fried LP, Tangen CM, Walston J et al (2001) Frailty in older adults: evidence for a phenotype. J Gerontol A Biol Sci Med Sci 56:M146-M156

6. Penninx BW, Ferrucci L, Leveille SG, Rantanen T, Pahor M, Guralnik JM (2000) Lower extremity performance in nondisabled older persons as a predictor of subsequent hospitalization. J Gerontol A Biol Sci Med Sci 55:M691-M697 
7. Guralnik JM, Simonsick EM, Ferrucci L et al (1994) A short physical performance battery assessing lower extremity function: association with self-reported disability and prediction of mortality and nursing home admission. J Gerontol 49:M85-M94

8. Guralnik JM, Ferrucci L, Simonsick EM, Salive ME, Wallace RB (1995) Lower-extremity function in persons over the age of 70 years as a predictor of subsequent disability. N Engl J Med 332:556-561

9. Guralnik JM, Ferrucci L, Pieper CF et al (2000) Lower extremity function and subsequent disability: consistency across studies, predictive models, and value of gait speed alone compared with the short physical performance battery. J Gerontol A Biol Sci Med Sci 55:M221-M231

10. Studenski S, Perera S, Wallace D et al (2003) Physical performance measures in the clinical setting. $\mathrm{J}$ Am Geriatr Soc $51: 314-322$

11. Pahor M, Blair SN, Espeland M et al (2006) Effects of a physical activity intervention on measures of physical performance: results of the lifestyle interventions and independence for Elders Pilot (LIFE-P) study. J Gerontol A Biol Sci Med Sci 61:1157-1165

12. Macchi C, Polcaro P, Cecchi F et al (2009) One-year adherence to exercise in elderly patients receiving postacute inpatient rehabilitation after cardiac surgery. Am J Phys Med Rehabil 88:727-734

13. Audelin MC, Savage PD, Ades PA (2008) Exercise-based cardiac rehabilitation for very old patients ( $>$ or $=75$ years): focus on physical function. J Cardiopulm Rehabil Prev 28:163-173

14. Leon AS, Franklin BA, Costa F et al (2005) Cardiac rehabilitation and secondary prevention of coronary heart disease: an American Heart Association scientific statement from the Council on Clinical Cardiology (Subcommittee on Exercise, Cardiac Rehabilitation, and Prevention) and the Council on Nutrition, Physical Activity, and Metabolism (Subcommittee on Physical Activity), in collaboration with the American association of Cardiovascular and Pulmonary Rehabilitation. Circulation 111:369-376

15. Lee DH, Buth KJ, Martin BJ, Yip AM, Hirsch GM (2010) Frail patients are at increased risk for mortality and prolonged institutional care after cardiac surgery. Circulation 121:973-978

16. Rosamond W, Flegal K, Furie K et al (2008) Heart disease and stroke statistics-2008 update: a report from the American Heart Association Statistics Committee and Stroke Statistics Subcommittee. Circulation 117:e25-e146
17. Folstein MF, Folstein SE, McHugh PR (1975) Mini-mental state A practical method for grading the cognitive state of patients for the clinician. J Psychiatr Res 12:189-198

18. Yesavage JA, Brink TL, Rose TL et al (1982) Development and validation of a geriatric depression screening scale: a preliminary report. J Psychiatr Res 17:37-49

19. Polcaro P, Lova RM, Guarducci L et al (2008) Left-ventricular function and physical performance on the 6-min walk test in older patients after inpatient cardiac rehabilitation. Am J Phys Med Rehabil 87:46-52

20. Macchi C, Fattirolli F, Lova RM et al (2007) Early and late rehabilitation and physical training in elderly patients after cardiac surgery. Am J Phys Med Rehabil 86:826-834

21. Kwon S, Perera S, Pahor M et al (2009) What is a meaningful change in physical performance? Findings from a clinical trial in older adults (the LIFE-P study). J Nutr Health Aging 13:538-544

22. Perera S, Mody SH, Woodman RC, Studenski SA (2006) Meaningful change and responsiveness in common physical performance measures in older adults. J Am Geriatr Soc 54:743-749

23. Lang RM, Bierig M, Devereux RB et al (2005) Recommendations for chamber quantification: a report from the American Society of Echocardiography's Guidelines and Standards Committee and the Chamber Quantification Writing Group, developed in conjunction with the European Association of Echocardiography, a branch of the European Society of Cardiology. J Am Soc Echocardiogr 18:1440-1463

24. ATS statement: guidelines for the six-minute walk test Am J Respir Crit Care Med 2002; 166:111-117

25. Moher D, Hopewell S, Schulz KF et al (2010) CONSORT 2010 explanation and elaboration: updated guidelines for reporting parallel group randomised trials. J Clin Epidemiol 63:e1-e37

26. Maillet JM, Somme D, Hennel E, Lessana A, Saint-Jean O, Brodaty D (2009) Frailty after aortic valve replacement (AVR) in octogenarians. Arch Gerontol Geriatr 48:391-396

27. Huber CH, Goeber V, Berdat P, Carrel T, Eckstein F (2007) Benefits of cardiac surgery in octogenarians-a postoperative quality of life assessment. Eur J Cardiothorac Surg 31:1099-1105

28. Gill TM, Allore HG, Holford TR, Guo Z (2004) Hospitalization, restricted activity, and the development of disability among older persons. JAMA 292:2115-2124 